\title{
Microbiological surveillance in patients with cystic fibrosis
}

\author{
Paola Gualdi', Roberta Gazzini', Michele Schinella', Ermanno Baldo², Grazia Dinella², Cristina Guerzoni ${ }^{2}$ \\ I Laboratorio Chimica clinica e microbiologia Ospedale S.Maria del Carmine Rovereto (TN) \\ 2 U.O. Pediatria - Servizio di supporto provinciale per la cura della Fibrosi Cistica Ospedale S.Maria del Carmine Rovereto (TN)
}

Key words: cystic fibrosis, pulmonary infections, microbiological surveillance

\section{Sorveglianza microbiologica in pazienti affetti da fibrosi cistica}

\section{SUMMARY}

Pulmonary infections in patients with cystic fibrosis (CF), are a major cause of morbidity and mortality. Prevention, diagnosis and therapy in cystic fibrosis, lead to the necessary collaboration between clinical and laboratory to identify effective strategies and appropriate solutions to address the problems inherent isolation micro-organisms, antibiotic strategies, overcoming of bacterial resistance and other problems management of these patients. The task of the microbiology laboratory and research in quickly and accurately, the agents responsible for these infectious processes, in order to isolate them from material, identify and determine their sensitivity antibiotics.

A microbiological surveillance on 34 patients ( 13 males and 21 females) with CF and related to the "Support Services Provincial Trento for the treatment of cystic fibrosis "in the period July 2005 - August 2008, was carried out.

180 Gram positive and 278 of Gram negative bacteria as well as 235 fungi wre collected. Staphylococcus aureus was the most frequently organism found in patients with CF with an incidence of $23 \%$ on 156 strains isolated, Pseudomonas aeruginosa was collected $19 \%$ of all microorganisms isolated corresponding to I3I strains, Candida albicans is the yeast often isolated with a frequency $22 \%$ equal to I 49 isolates, Aspergillus fumigatus was isolated at a rate of $8 \%$.

From the data we collected and processed has been noted that the local epidemiology of CF patients reflects as reported in the scientific literature and national international consulting, both as a type microorganisms that frequency also isolated compared to age groups. Considering the score of Bartlett as discriminating respiratory fitness of the material, it has been observed that only 32 samples over 327 total $(10 \%)$ would materials insignificant. It follows therefore that the time of sample collection, followed by personnel (physiotherapists dedicated to CF patients), represents a crucial step in guarantee the quality of the preanalytical phase.

La Fibrosi Cistica (FC) o mucoviscidosi è una malattia congenita, cronica, evolutiva, trasmessa con meccanismo autosomico recessivo più frequente nella popolazione caucasica, con un' incidenza che varia da 1:2500 a 1:2700 $(2,5)$.

La FC è una malattia genetica multisistemica caratterizzata, nella sua forma classica, da un esordio in epoca neonataleinfantile e da un quadro che comprende ipersalinità del sudore, insufficienza pancreatica esocrina grave e progressiva broncopneumopatia cronica $(4,6)$.

Nelle regioni Veneto e Trentino-Alto Adige, le quali attuano un programma di screening neonatale di massa, l'incidenza osservata è di 1:2600 nati vivi e di 1:27 per quanto riguarda i portatori sani (eterozigoti) (3).

Le infezioni polmonari, in pazienti affetti da fibrosi cistica, rappresentano una delle principali cause di morbidità e mortalità. La prevenzione, la diagnosi e la terapia in fibrosi cistica, portano alla necessaria collaborazione tra clinica e laboratorio per poter individuare strategie efficaci e soluzioni appropriate per affrontare le problematiche inerenti l'isolamento dei microrganismi, le strategie antibiotiche, il superamento delle resistenze batteriche e altri problemi legati alla gestione di questi pazienti $(1,7)$.

Compito del laboratorio di microbiologia è ricercare, in modo rapido ed accurato, gli agenti responsabili di questi processi infettivi, con lo scopo di isolarli dal materiale, identificarli e determinare la loro sensibilità agli antibiotici. È stata effettuata una sorveglianza microbiologica su 34 pazienti (13 maschi e 21 femmine) affetti da FC ed afferenti al "Servizio di supporto provinciale di Trento per la cura della fibrosi cistica” nel periodo luglio 2005-agosto 2008. Il Laboratorio di Rovereto per l'esame dei materiali provenienti dalle basse vie respiratorie di pazienti affetti da FC quali espettorato, tampone faringeo profondo, aspirato ipofaringeo utilizza il seguente protocollo: esame microscopico; preparazione del campione; esame batteriologico colturale; identificazione; antibiogramma.

I campioni di materiale respiratorio di pazienti con FC, vengono seminati sui seguenti terreni: agar Columbia contenente acido nalidixico; Tryptone Soy Agar; agar cioccolato addizionato con bacitracina; agar MacConkey; Mannitol Salt Agar; bile esculina azide agar; Sabouraud Dextrose Agar con cloramfenicolo; Burkholderia cepacia agar.

L'identificazione e l'antibiogramma vengono eseguiti con sistema Vitek 2 e MiniApi (bioMérieux) ed il controllo di qualità interno con ceppi ATCC (E.coli 25922, P.aeruginosa 27853, S.aureus 29213, E.faecalis 29212, S.pneumoniae 49619, H.influenzae 49247) confrontando i dati di riferimento indicati e aggiornati periodicamente dal CLSI.

$\grave{E}$ stato eseguito uno studio generale sulla frequenza di ogni singolo microrganismo isolato e riscontrato in periodi diversi nei pazienti con FC per un totale di 693 ceppi comprendenti sia batteri che funghi. Sono stati riscontrati 180 ceppi di batteri Gram positivi, 278 ceppi di batteri Gram negativi e 235 ceppi di miceti. È emerso che $S$. aureus è il microrganismo più volte isolato in pazienti con FC con una frequenza del $23 \%$ pari a 156 ceppi isolati; P.aeruginosa è stato riscontrato nel $19 \%$ di tutti i microrganismi isolati pari a 131 ceppi; C. albicans è il lievito più volte isolato con una frequenza del $22 \%$ pari a 149 ceppi isolati; A. fumigatus è stato isolato 54 volte pari ad una frequenza dell'8\% (Tabella 1).

È stato inoltre condotto uno studio sui microrganismi maggiormente riscontrati a seconda dell'età del paziente, considerando che esiste un preciso pattern batterico età-specifico responsabile della malattia polmonare in FC (Tabella 2).

Dai dati da noi raccolti e rielaborati si è potuto notare che l'epidemiologia locale dei pazienti con FC rispecchia quanto riportato nella letteratura scientifica nazionale e internazionale consultata, sia come tipologia di microrganismi che di frequenza di isolamenti anche rispetto alle fasce d'età.

Considerando lo score di Bartlett come discriminante di idoneità del materiale respiratorio, si è potuto osservare che solo 32 campioni sui 327 totali (10\%) sarebbero materiali poco significativi. Si evince quindi che il momento della raccolta del campione, seguita da personale specializzato (fisioterapista dedicata a pazienti con FC), rappresenta un passaggio cruciale a garanzia della qualità della fase preanalitica.

\section{Corresponding author: Paola Gualdi}

Laboratorio Chimica clinica e microbiologia Ospedale S. Maria del Carmine Rovereto (TN)

38068 Rovereto (TN) - P.le S.Maria, 6 - Tel. 0464403489 - Fax 0464403154

E-mail: paola.gualdi@apss.tn.it 
Tabella I. Totale microrganismi isolati (totale 693 ceppi).

\begin{tabular}{|l|c|c|}
\hline \multicolumn{1}{|c|}{ Microrganismo } & $\begin{array}{c}\mathbf{n}^{\circ} \text { di volte } \\
\text { isolato }\end{array}$ & Percentuale \\
\hline Altri microrganismi (n 27) & 39 & $6 \%$ \\
\hline $\begin{array}{l}\text { Achromobacter } \text { xylosoxidans spp } \\
\text { xylosoxidans }\end{array}$ & 29 & $4 \%$ \\
\hline Aspergillus flavus & 4 & $1 \%$ \\
\hline Aspergillus fumigatus & 54 & $8 \%$ \\
\hline Burkholderia cepacia & 25 & $4 \%$ \\
\hline Candida albicans & 149 & $22 \%$ \\
\hline Candida famata & 6 & $1 \%$ \\
\hline Candida species, not albicans & 11 & $2 \%$ \\
\hline Enterococcus faecalis & 15 & $2 \%$ \\
\hline Haemophilus influenzae & 38 & $5 \%$ \\
\hline Pseudomonas aeruginosa & 131 & $19 \%$ \\
\hline Pseudomonas fluorescens & 4 & $1 \%$ \\
\hline Staphylococcus aureus & 156 & $23 \%$ \\
\hline $\begin{array}{l}\text { Stenotrophomonas } \\
\text { maltophilia }\end{array}$ & 26 & $4 \%$ \\
\hline Streptococcus agalactiae & 693 & $1 \%$ \\
\hline ToTALE & & $100 \%$ \\
\hline
\end{tabular}

Tabella 2. Numero di microrganismi isolati per fascia d'età.

\begin{tabular}{|l|c|c|c|}
\hline & \multicolumn{3}{|c|}{$\mathbf{n}^{\circ}$ di isolati per singolo microrganismo } \\
\hline Microrganismo & $\begin{array}{c}\text { Età Pediatrica } \\
\mathbf{0 - 1 4} \text { anni }\end{array}$ & $\begin{array}{c}\text { Età } \\
\text { Adolescenziale } \\
\mathbf{1 5 - 2 0} \text { anni }\end{array}$ & $\begin{array}{c}\text { Età Adulta } \\
>\mathbf{2 0} \text { anni }\end{array}$ \\
\hline S. aureus & 51 & 22 & 83 \\
\hline H. influenzae & 31 & 3 & 4 \\
\hline P. aeruginosa & 29 & 6 & 96 \\
\hline S. maltophilia & 19 & 4 & 3 \\
\hline A. xylosoxidans & 4 & 0 & 25 \\
\hline B. cepacia & 0 & 0 & 25 \\
\hline A.spergillus spp. & 20 & 4 & 34 \\
\hline Candida spp. & 47 & 27 & 100 \\
\hline
\end{tabular}

\section{BIBLIOGRAFIA}

1. Campana S, Favari F, Manno G, et al. Fibrosi cistica e microbiologia. Orizzonti FC. 2007; 3(2): 2-28.

2. Cao A, Dallapiccola B, Notarangelo LD. Malattie genetiche geni e molecule diagnosi, prevenzione e terapia. Piccin 2004; 195-227.

3. Castellani C, Bonizzato A, Cabrini G, Mastella G. Newborn screening strategy: a field study in an area with high allelic heterogeneity. Acta Paediatr 1997; 86: 497-502.

4. http://www.fibrosicistica.it/page.php?cPath $=0 \quad 79$

5. Lewis MJ, Lewis EH, Amos JA, Tsongalis GJ. Cystic fibrosis. Am J Clin Pathol 2003; 120 Suppl: S3-13.

6. Stern RC. The diagnosis of cystic fibrosis. N Engl J Med 1997; 336:487491.

7. Yankaskas JR, Marshall BC, Surfian B, Simon RH, Rodman D. Cystic fibrosis adult care: consensus conference report. Chest 2004; 125(1 Suppl): 1S-39S. 